\title{
Uniformly Primal Submodule over Noncommutative Ring
}

\author{
Lamis J. M. Abulebda $(\mathbb{D}$ \\ Department of Mathematics, College of Arts and Sciences, University of Balamand Dubai, Dubai, UAE
}

Correspondence should be addressed to Lamis J. M. Abulebda; lamis_jomah@yahoo.com

Received 14 July 2020; Revised 31 August 2020; Accepted 30 September 2020; Published 22 October 2020

Academic Editor: Andrei V. Kelarev

Copyright (C) 2020 Lamis J. M. Abulebda. This is an open access article distributed under the Creative Commons Attribution License, which permits unrestricted use, distribution, and reproduction in any medium, provided the original work is properly cited.

\begin{abstract}
Let $R$ be an associative ring with identity and $M$ be a unitary right $R$-module. A submodule $N$ of $M$ is called a uniformly primal submodule provided that the subset $B$ of $R$ is uniformly not right prime to $N$, if there exists an element $s \in M-N$ with $\operatorname{sRB} \subseteq N$.The set $\operatorname{adj}(N)=\{r \in R \mid t \mathrm{mRr} n \subseteq q N h$ for Some $m \in C M\}$ is uniformly not prime to $N$.This paper is concerned with the properties of uniformly primal submodules. Also, we generalize the prime avoidance theorem for modules over noncommutative rings to the uniformly primal avoidance theorem for modules.
\end{abstract}

\section{Introduction}

Throughout this paper, all rings are associative with identity and all modules are unitary modules. For detailed description regarding rings and modules, interested readers are encouraged to go through the book of Kelarev et al. [1]. The concept of uniformly primal ideal has been introduced and studied by Barnes [2]. Let $A$ be an ideal of $R$. The ideal $B$ of $R$ is uniformly not right prime to $A$, if there exists an element $y \in R-A$ with $\mathrm{yRB} \subseteq A$. $A$ is called uniformly primal if $\operatorname{adj}(A)$ is uniformly not right prime to $\mathrm{A}$ where $\operatorname{adj}(A)=\{x \in R \mid y R x \subseteq A$ for some $y \in R-A\}$. The prime avoidance theorem for rings with identity [3] states that if an ideal $I$ of a ring is contained in a union of a finite number of prime ideals $\left(P_{1}, P_{2}, \ldots, P_{n}\right)$, then $I$ must be contained in $P_{k}$ for some $k \in\{1,2, \ldots, n\}$. Karamzadeh [4] generalizes the prime avoidance theorem for any ring that is not necessarily commutative. The aim of Section 1 is to generalize the prime avoidance theorem for rings over noncommutative rings to the uniformly primal avoidance theorem over noncommutative rings.

The concept of uniformly primal submodules has been introduced and studied by Dauns in [5]. A submodule $N$ of $M$ is called a uniformly primal submodule provided that the set $\operatorname{adj}(N)=\{x \in R \mid \operatorname{mRr} \subseteq N$ for some $m \in M\}$ is uniformly not prime to $\mathrm{N}$, where the subset $\mathrm{B}$ of $R$ is uniformly not right prime to $N$ if there exists an element $s \in M-N$ with $s R B \subseteq N$. In particular, a number of papers concerning primal submodules have been studied by various authors (see, for example, [6-10]). In Section 2, we give some basic results about uniformly primal submodules and show that $N_{1}, N_{2}, \ldots, N_{n}$ is a finite collection of uniformly primal submodules of an $R$-module $M$ with $\operatorname{adj}\left(N_{j}\right)=P_{j}$ for every $j$ and $\quad\left(N_{j}: M\right) \not \subset \operatorname{adj}\left(N_{k}\right)$ whenever $j \neq k$. Then, $S^{\prime}=M-\cup_{i=1}^{n} N_{i}$ is an S-system subset of $M$, where $S=R-\cup_{i=1}^{n} P_{i}$. Also, we study the prime avoidance theorem for modules over noncommutative rings and generalize it to the uniformly primal avoidance theorem for modules.

\section{Uniformly Primal Ideal}

The concept of primal ideals over noncommutative has been introduced and studied by Fuchs [11].

Definition 1. Let A be an ideal of $R$. The adjoint of $A$ is the set of all elements of $R$ that are not right prime to $A$ and denoted by adj $(A)$. In other words, $\operatorname{adj}(A)=\{x \in R \mid y R x \subseteq A$ for some $y \in R-A\}$.

Definition 2. The ideal $A$ of $R$ is said to be primal if $\operatorname{adj}(A)$ forms an ideal of $R$. In this case, the adjoint of $A$ will also be called the adjoint ideal of $A$. 
Definition 3. The ideal $B$ of $R$ is uniformly not right prime to $A$ if there exists an element $y \in R-A$ with $\mathrm{yRB} \subseteq A$.

Definition 4. An ideal $A$ of a ring $R$ is said to be uniformly primal if $\operatorname{adj}(A)$ is uniformly not right prime to $A$.

Proposition 1 (see [2]). If $A$ is a uniformly primal ideal in $R$, then $\operatorname{adj}(A)$ is a prime ideal of $R$.

Proposition 2 (see [3]). If $P, P_{1}$, and $P_{2}$ are ideals of $R$ such that $P \subseteq P_{1} \cup P_{2}$, then either $P \subseteq P_{1}$ or $P \subseteq P_{2}$. The definition of efficient union of ideals was introduced in the rings that are commutative (see [12]). We give a generalization to it in rings that are not necessary commutative as follows.

Definition 5. Let $P, P_{1}, P_{2}, \ldots, P_{s}$ be ideals of a ring $R$. The covering $P \subseteq P_{1} \cup P_{2} \cup \cdots \cup P_{s}$ of $P$ is called efficient if $P$ is not contained in the union of any $s-1$ of the ideals $P_{i}^{\prime} s$. Analogously, we shall say $P=P_{1} \cup P_{2} \cup \cdots \cup P_{s}$ is an efficient union if none of the $P_{i}^{\prime} s$ may be excluded. Any cover or union consisting of ideals of $R$ can be reduced to an efficient one, called an efficient reduction, by deleting any unnecessary terms. The following very important lemma is based on McCoy over commutative rings (see [3]).

Lemma 1 (see [3]). Let $P=P_{1} \cup P_{2} \cup \cdots \cup P_{s}$ be an efficient union of ideals where $s>2$. Then, $\cap_{j \neq k} P_{j}=\cap_{j=1}^{s} P_{j}$ for all $k \in\{1,2, \ldots, s\}$. As an application, we obtain following corollary.

Corollary 1. Let $P \subseteq P_{1} \cup P_{2} \cup \cdots \cup P_{s}$ be an efficient cover of ideals of a ring $R$ where $s>2$. Then, $P \cap \cap_{j \neq k} P_{j} \subseteq P_{k}$ for all $k \in\{1,2, \ldots, s\}$.

Proof. Since $P \subseteq P_{1} \cup P_{2} \cup \cdots \cup P_{s}$ is an efficient covering, $P \subseteq\left(P \cap P_{1}\right) \cup\left(P \cap P_{2}\right) \cup \cdots \cup\left(P \cap P_{s}\right)$ is an efficient union. Now, by Lemma $1, P \cap \cap{ }_{j \neq k} P_{j}=\cap_{j \neq k}\left(P \cap P_{j}\right) \subseteq P_{k}$. To prove the uniformly primal avoidance theorem for rings, we need the following result on the uniformly primal ideal.

Proposition 3. Let $P \subseteq P_{1} \cup P_{2} \cup \cdots \cup P_{s}$ be an efficient covering consisting of ideals where $s>2$. If adj $\left(P_{i}\right) \not \subset \operatorname{adj}\left(P_{k}\right)$ for every $i \neq j$, then no $P_{k}$ for $k \in\{1,2, \ldots, s\}$ is a uniformly primal ideal of $R$.

Proof. Suppose that some $P_{k}$ is uniformly primal ideal. Since $P \subseteq P_{1} \cup P_{2} \cup \cdots \cup P_{s}$ is an efficient covering, there exists an element $a_{k} \in P-P_{k}$. If $i \neq j$, then $\operatorname{adj}\left(P_{i}\right) \not \subset \operatorname{adj}\left(P_{k}\right)$, so there exists $r_{j} \in \operatorname{adj}\left(P_{j}\right)$ such that $r_{j} \notin \operatorname{adj}\left(P_{k}\right)$. Since $P_{k}$ is a uniformly primal ideal, then by Proposition $1, \operatorname{adj}\left(P_{k}\right)$ is a prime ideal of $R$. Therefore, $r=\prod_{i \neq k} r_{i} \in \operatorname{adj}\left(P_{j}\right)$, but $r \notin \operatorname{adj}\left(P_{k}\right)$. Consequently, $e_{k} \operatorname{Rr} \subseteq P \cap P_{j}$ for every $k \neq j$, but $e_{k} \operatorname{Rr} \not \subset P_{k}$, which contradicts the fact that $P \cap \cap{ }_{j \neq k} P_{j}=\cap j \neq k\left(P \cap P_{j}\right) \subseteq P_{k}$ (by Corollary 1$)$. Therefore, no $P_{k}$ is a uniformly primal. Now, we will give the proof of the main theorem of this section.
Theorem 1 (uniformly primal avoidance theorem of rings). Let $P_{1}, P_{2}, \ldots, P_{s}$ be a finite number of ideals of a ring $R$ and $P$ be an ideal of $R$ such that $P \subseteq P_{1} \cup P_{2} \cup \cdots \cup P_{s}$. Assume that at least two of the $P_{i}^{\prime} s$ are not uniformly primal and that $\operatorname{adj}\left(P_{i}\right) \not \subset \operatorname{adj}\left(P_{j}\right)$ whenever $i \neq j$. Then, $P \subseteq P_{k}$ for some $k \in\{1,2, \ldots, s\}$.

Proof. For the given covering $P \subseteq P_{1} \cup P_{2} \cup \cdots \cup P_{s}$, let $P \subseteq P_{\alpha_{1}} \cup P_{\alpha_{2}} \cup \cdots \cup P_{\alpha_{m}}$ be its efficient reduction. Then, $1 \leq m \leq s$ and $m \neq 2$ where if $m=2$, then by Proposition 2, $P \subseteq P_{1}$ or $P \subseteq P_{2}$. If $m>2$, then there exists at least one $P_{\alpha_{r}}$ to be uniformly primal ideal. By Proposition 3 , this is impossible as $\operatorname{adj}\left(P_{i}\right) \not \subset \operatorname{adj}\left(P_{j}\right)$ if $i \neq j$. Hence, $m=1$, so $P \subseteq P_{k}$ for some $k$.

\section{Uniformly Primal Submodule}

The concept of primal submodules has been introduced and studied by Dauns in [5].

Definition 6. Let $M$ be an $R$-module and $N$ be a submodule of $M$. For any $a \in R$, the submodule $\{m \in M \mid m R a \subseteq N\}$ is denoted by $N a^{-1}$. Analogously, for a subset $A$ of $R, N A^{-1}$ $=\{m \in M \mid \mathrm{mRA} \subseteq N\}$ where $\mathrm{RA}=\{$ mra: $\mid r \in R, a \in A\}$.

Definition 7. Let $M$ be an $R$-module and $N$ be a submodule of $M$. The element $a \in R$ is right prime to $\mathrm{N}$ if $N a^{(-1)}=N$, i.e., if $\mathrm{mRa} \subseteq N$ where $m \in M$ implies $m \in N$. The element $a \in R$ is not right prime to $\mathrm{N}$ if $N a^{(-1)} \neq N$, i.e., there exists an element $m \in M-N$ with $\mathrm{mRa} \subseteq N$, since $N \subseteq N a^{(-1)}$. A subset $A$ of $R$ is not right prime to $N$ if for any $a \in A, a$ is not right prime to $\mathrm{N}$. In this case, we say that $A$ is pointwise not right prime to $N$. The subset $A$ of $R$ is uniformly not right prime to $N$ if there exists an element $s \in M-N$ with sRA $\subseteq N$, i.e., $A$ is uniformly not right prime to $N$ if and only if $\mathrm{NA}^{-1} \neq N$.

Definition 8. Let $M$ be an $R$-module and $N$ be a submodule of $M$. The adjoint of $N$ is the set of all elements of $R$ that are not right prime to $N$ and denoted by $\operatorname{adj}(N)$. In the other words, $\operatorname{adj}(N)=\{r \in R \mid \mathrm{mRr} \subseteq N$ forsome $m \in M\}$.

Definition 9. Let $M$ be an $R$-module. A proper submodule $N$ of $M$ is said to be primal if adj $(N)$ forms an ideal of $R$. In this case, the adjoint of $N$ will also be called the adjoint ideal of $N$.

Proposition 4. Let $N$ be a submodule of an $R$-module $M$. If $\operatorname{adj}(N)$ is uniformly not prime to $N$, then adj $(N)$ is an ideal of $R$, and as a consequence, $N$ is a primal submodule.

Definition 10. Let $M$ be an $R$-module. A proper submodule $N$ of $M$ is said to be uniformly primal if adj $(N)$ is uniformly not prime to $\mathrm{N}$.

Proposition 5 (see [8]). Let $M$ be an R-module. If $N$ is a uniformly primal submodule of $M$, then $\operatorname{adj}(N)$ is a prime ideal of $R$. In the following propositions, we show the behavior of a primal submodule under isomorphism. 
Proposition 6. Let $f: M \longrightarrow M^{\prime}$ be a module isomorphism. If $N$ is a primal submodule of $M$, then $f(\operatorname{adj}(N))=\operatorname{adj}(f(N))$.

Proof. Let $a \in f(\operatorname{adj}(N))$. Since $f$ is a module isomorphism, then $f^{-1}(a) \in \operatorname{adj}(N)$ so that there exist $s \in M-N$ such that $\operatorname{sRf}^{-1}(a) \subseteq N$. Since $f$ is a module isomorphism, then $f(s) \mathrm{Ra}=f\left(\mathrm{sRf}^{-1}(a)\right) \subseteq f(N)$, but $s \in M-N$ so that $f(s) \in M^{\prime}-f(N)$ which implies that $a \in \operatorname{adj}(f(N))$. Thus, $f(\operatorname{adj}(N)) \subseteq \operatorname{adj}(f(N))$. Now, let $a \in f(\operatorname{adj}(N))$ so that there exist $t \in M^{\prime}-f(N)$ such that $\operatorname{Ra} \subseteq f(N)$. Since $f$ is a module isomorphism, then $f^{-1}(t) \mathrm{Rf}^{-1}(a) \subseteq$ $f(N)=f^{-1}(\mathrm{tRa}) \subseteq f^{-1}(f(N))=N$, but $t \in M^{\prime}-f(N)$ so that $f^{-1}(t) \in M-N$ which implies that $f^{-1}(a) \in \operatorname{adj}(N)$. That is, $a \in f(\operatorname{adj}(N))$. Thus, $f(\operatorname{adj}(N)) \subseteq \operatorname{adj}(f(N))$.

Proposition 7. Let $f: M \longrightarrow M^{\prime}$ be a module isomorphism. If $N$ is a primal submodule of $M$, then $f(N)$ is a primal submodule of $M^{\prime}$.

Proof. Let $a, b \in(\operatorname{adj}(f(N)))$. Since $f$ is a module isomorphism, then by Proposition 6, we have $f^{-1}(a)$, $f^{-1}(b) \in f^{-1}(\operatorname{adj}(f(N)))=\operatorname{adj}\left(f^{-1}(f(N))\right)=\operatorname{adj}(N)$.

But $N$ is a primal submodule; then, $f^{-1}(a+b)=$ $f^{-1}(a)+f^{-1}(b) \in \operatorname{adj}(N)$. Also, since $f$ is a module isomorphism, we have $a+b \in f(\operatorname{adj}(N))$. Hence, $a+b \in \operatorname{adj}(f(N))$ by Proposition 6 , where $f(N)$ is a primal submodule of $M^{\prime}$.

Proposition 8. Let $N$ and $L$ be proper submodules of an $R$-module $M$ and $I$ be an ideal of $R$. If $L I \subseteq N$, then either $L \subseteq N$ or $I \subseteq \operatorname{adj}(N)$.

Proof. Assume $L \not \subset N$; then, there is $l \in L-N$. For each $a \in I$, lRa $\subseteq$ LI $\subseteq N$ while $l \notin N$; thus, $a \in \operatorname{adj}(N)$. Callialp and Takir introduced the following definition (see [13]).

Definition 11. Let $N, N_{1}, N_{2}, \ldots, N_{n}$ be submodules of an $R$-module $M$. The covering $N \subseteq N_{1} \cup N_{2} \cup \cdots \cup N_{n}$ of $N$ is called efficient if $N$ is not contained in the union of any $n-1$ of the submodules $N_{i}^{\prime} s$. Analogously, we shall say $N \subseteq N_{1} \cup N_{2} \cup \cdots \cup N_{n}$ is an efficient union if none of the $N_{i}^{\prime} s$ may be excluded.

Proposition 9. If $L$ and $N$ are submodules of an $R$-module $M$, then $(L \cap N: M)=(L: M) \cap(N: M)$.

Proof. Let $a \in(L \cap N: M)$. Then, $M a \subseteq L \cap N$. Thus, $M a \subseteq L$ and $M a \subseteq N$; hence, $a \in(L: M) \cap(N: M)$. Therefore, $(L \cap N: M) \subseteq(L: M) \cap(N: M)$. Now, if $b \in(L: M) \cap$ $(N: M)$, then $b \in(L: M)$ and $b \in(N: M)$ so that $M b \subseteq L$ and $\mathrm{Mb} \subseteq N$; thus, $\mathrm{Mb} \subseteq L \cap N$ and then $b \in(L \cap N: M)$. Therefore, $(L: M) \cap(N: M)(L \cap N: M)$.

Proposition 10 (see [13]). Let $N \subseteq N_{1} \cup N_{2} \cup \cdots \cup N_{n}$ be an efficient cover of submodules of an R-module $M$ where $n>2$. Then, $N \cap \cap{ }_{j \neq k} N_{j} \subseteq N_{k}$ for all $k \in\{1,2, \ldots, n\}$. Now, by using Propositions 9 and 10 we will prove the following lemma and theorem.
Lemma 2. Let $N \subseteq N_{1} \cup N_{2} \cup \cdots \cup N_{n}$ be an efficient cover of submodules of an R-module $M$ where $n>2$; then, for all $j \in\{1,2, \ldots, n\}, \cap_{i \neq j}\left(N_{i}: M\right) \subseteq \operatorname{adj}\left(N_{j}\right)$.

Proof. Let $j \in\{1,2, \ldots, n\}$. Put $\cap_{i \neq j}\left(N_{i}: M\right)=P_{j}$. By Proposition 9, $\left(\cap_{i \neq j} N_{i}: M\right)=P_{j}$. So, $\mathrm{MP}_{j} \subseteq \cap_{i \neq j} N_{i}$, and thus $\mathrm{NP}_{j} \subseteq \cap_{i \neq j} N_{i}$. But $\mathrm{NP}_{j} \subseteq N$; then, $N P_{j} \subseteq N \cap$ $\left(\cap_{i \neq j} N_{i}\right) \subseteq N_{j}$ by Proposition 10. This implies either $N \subseteq N_{j}$ or $P_{j} \subseteq \operatorname{adj}\left(N_{j}\right)$. But $N \not \subset N_{j}$, and this implies that $\cap_{i \neq j}\left(N_{i}: M\right) \subseteq \operatorname{adj}\left(N_{j}\right)$.

Theorem 2. Let $N$ be a submodule of an R-module $M$. If $N_{1}, N_{2}, \ldots, N_{n}$ are submodules of $M$ such that $N \subseteq N_{1} \cup N_{2} \cup \cdots \cup N_{n}$ and $\cap_{i \neq j}\left(N_{i}: M\right) \not \subset a d j\left(N_{j}\right)$ for all $j=1,2, \ldots, n$ except possibly for at most two of the $j$ 's, then $N \subseteq N_{k}$ for some $k \in\{1,2, \ldots, n\}$.

Proof. For the given covering $N \subseteq N_{1} \cup N_{2} \cup \cdots \cup N_{n}$, let $N \subseteq N_{\alpha_{1}} \cup N_{\alpha_{2}} \cup \cdots \cup N_{\alpha_{m}}$ be its efficient reduction. Then, $1 \leq m \leq n$ and $m \neq 2$. If $m>2$, then there exists at least one $N_{i_{j}}$ satisfying $\cap_{i \neq j}\left(N_{\alpha_{i}}: M\right) \subseteq \operatorname{adj}\left(N_{\alpha_{j}}\right)$ which is contradiction to Lemma 2. Hence, $m=1$, so $N \subseteq N_{\alpha_{1}}=N_{k}$ for some $k \in\{1,2, \ldots, n\}$.

Bland in [14] proved the following result.

Corollary 2. Let $P$ be a prime ideal of a ring $R$ and suppose that $A$ and $B$ are ideals in $R$. If $A \cap B \subseteq P$, then either $A \subseteq P$ or $B \subseteq P$. The following corollary follows immediately from Proposition 5 and Corollary 2.

Corollary 3. Let $N_{1}, N_{2}, \ldots, N_{n}$ be uniformly primal submodules. Then, the following two conditions are equivalent:

(a) $\cap_{i \neq j}\left(N_{i}: M\right) \not \subset \operatorname{adj}\left(N_{j}\right)$

(b) $\left(N_{i}: M\right) \not \subset \operatorname{adj}\left(N_{j}\right)$ whenever $i \neq j$

Now, the main theorem of this section is uniformly primal avoidance theorem which follows immediately from Proposition 5, Theorem 2, and Corollary 3.

Theorem 3 (uniformly primal avoidance theorem for modules). Let $N$ be a submodule of an R-module $M$. $N_{1}, N_{2}, \ldots, N_{n}$ are submodules of $M$ such that $N \subseteq N_{1} \cup N_{2} \cup \cdots \cup N_{n}$. Assume that at most two of the $N_{i}^{\prime} s$ are not uniformly primal and $\left(N_{i}: M\right) \not \subset \operatorname{adj}\left(N_{j}\right)$ whenever $i \neq j$; then, $N \subseteq N_{k}$ for some $k \in\{1,2, \ldots, n\}$.

Corollary 4 (see [5]). Let $M$ be an R-module. If $N$ is a prime submodule of $M$, then $N$ is primal. Since every prime submodule is uniformly primal by Corollary 4, then the uniformly primal avoidance theorem is a generalization of the prime avoidance theorem for modules. Now, we will recall the concept of S-system subsets of modules, which was introduced in [11] (also see $[13,15])$. Then, we will prove some results on the S-system and uniformly primal submodule.

Definition 12. A nonempty subset $S$ of a ring $R$ is said to be an $m$-system if for any $a, b \in S$, there exists $r \in R$ such that $\operatorname{arb} \in S$. 
Definition 13. Let $M$ be an $R$-module and $\mathrm{S}$ be an $\mathrm{m}$-system. A nonempty subset $\mathrm{N}$ of $R$-module $M$ is said to be a $S$-system if for any $a \in S$ and $m \in N$, there exists $r \in R$ such that mra $\in N$.

Proposition 11. Let $M$ be an $R$-module and $N$ be a uniformly primal submodule of $M$. Then, $M-N$ is an S-system where $S=R-\operatorname{adj}(N)$.

Proof. Since $\mathrm{N}$ is a uniformly primal submodule of $M$, by Proposition $5, \operatorname{adj}(N)$ is a prime ideal of R. Let $a \in S=$ $R-\operatorname{adj}(N)$ and $t \in M-N$, so $t \operatorname{Ra} \not \subset N$. Therefore, $M-N$ is an S-system.

Proposition 12. Let $P_{1}, P_{2}, \ldots, P_{n}$ be a finite number of prime ideals in a ring $R$ and $S=R-\cup_{i=1}^{n} P_{i}$. Then, $S$ is an $m$-system subset of $R$.

Proof. Let $a, b \in S$ and assume on the contrary that $\mathrm{aRb} \not \subset S$; thus, $\mathrm{aRb} \subseteq \cup_{i=1}^{n} P_{i}$. Then, $\mathrm{RaRbR} \subseteq \cup_{i=1}^{n} P_{i}$. Hence, by the prime avoidance theorem for rings (see [3] and [4]), we have $(\mathrm{RaR})(\mathrm{RbR})=\mathrm{RaRbR} \subseteq P_{i}$ for some $i=1,2, \ldots, n$. Since $P_{i}$ is prime, then either $\operatorname{RaR} \subseteq P_{i}$ or $\mathrm{RbR} \subseteq P_{i}$. If $\mathrm{RaR} \subseteq P_{i}$, then $a \notin S$ which is a contradiction. Similarly, if $\operatorname{RbR} \subseteq P_{i}$, then there exists $r \in R$ such that arb $\in S$. Therefore, $S$ is an $m$-system subset of $R$.

Proposition 13. Let $N_{1}, N_{2}, \ldots, N_{n}$ be a finite collection of uniformly primal submodules of an $R$-module $M$ with $\operatorname{adj}\left(N_{j}\right)=P_{j}$ for every $j$ and $\left(N_{j}: M\right) \not \subset \operatorname{adj}\left(N_{k}\right)$ whenever $j \neq k$. Then, $S^{*}=M-\cup_{i=1}^{n} N_{i}$ is an $S$-system subset of $M$, where $S=R-\cup_{i=1}^{n} P_{i}$.

Proof. By Proposition 12, $\mathrm{S}$ is an m-system subset of $R$, so to prove $S^{*}$ is an S-system, let $a \in S$ and $m \in S^{*}$ and assume on the contrary that $\operatorname{mRa} \not \subset S^{*}$; thus, $\operatorname{mRa} \subseteq \cup_{i=1}^{n} N_{i}$. Then, $\operatorname{mRaR} \subseteq \cup \cup_{i=1}^{n} N_{i}$, so by Theorem 3 (uniformly primal avoidance theorem for modules), we have $\mathrm{mRaR} \subseteq N_{i}$ and $\mathrm{mRa} \subseteq N_{i}$ for some $i=1,2, \ldots, n$. Since $N_{i}$ is uniformly primal, then $m \in N_{i}$ or $a \in \operatorname{adj}\left(N_{i}\right)$. If $m \in N_{i}$, then $m \notin S^{*}$ which is a contradiction. If $a \in \operatorname{adj}\left(N_{i}\right)$, then $a \notin S$. Then, there exists $r \in R$ such that mra $\in S^{*}$. Therefore, $S^{*}$ is an S-system subset of $M$.

\section{Data Availability}

No data were used to support this study.

\section{Conflicts of Interest}

The author declares that there are no conflicts of interest.

\section{References}

[1] A. V. Kelarev, R. Göbel, K. Rangaswamy, P. Schultz, and C. Vinsonhaler, Abelian Groups, Rings and Modules, American Mathematical Society, Providence, RI, USA, 2001.

[2] W. E. Barnes, "Primal ideals and isolated components in noncommutative rings," Transactions of the American Mathematical Society, vol. 82, no. 1, 16 pages, 1956.
[3] N. H. McCoy, "A note on finite unions of ideals and subgroups," Proceedings of the American Mathematical Society, vol. 8, no. 4, 633 pages, 1957.

[4] O. A. S. Karamzadeh, "The prime avoidance lemma revisited," Kyungpook Mathematical Journal, vol. 52, no. 2, pp. 149-153, 2012.

[5] J. Dauns, "Primal modules," Communications in Algebra, vol. 25 , no. 8 , pp. $2409-2435,1997$.

[6] N. J. Groenewald and D. Ssevviiri, "2-primal modules," Journal of Algebra and Its Applications, vol. 12, no. 05, Article ID 1250226, 2013.

[7] D. Ssevviiri, "A relationship between 2-primal modules and modules that satisfy the radical formula," International Electronic Journal of Algebra, vol. 18, no. 18, 34 pages, 2015.

[8] S. E. Atani and A. Y. Darani, "Some remarks on primal submodules," Sarajevo Journals of Mathematics, vol. 4, no. 17, pp. 181-190, 2008.

[9] A. A. Jaber, "Weakly primal graded superideals," Tamkang Journal of Mathematics, vol. 43, no. 1, pp. 123-135, 2012.

[10] N. D. H. Nghiem, S. Baupradist, and R. Chinram, "On nearly prime submodules of unitary modules," Journal of Mathematics, vol. 2018, Article ID 7202590, 4 pages, 2018.

[11] L. Fuchs, "On primal ideals," Proceedings of the American Mathematical Society, vol. 11 page, 1950.

[12] S. McAdam, "Finite coverings by ideals, ring theory," in Proceedings of Oklahoma Conference, pp. 163-171, Dekker, NY, USA, March 1973.

[13] F. Callialp and U. Tekir, "On finite union of prime submodules, Pakistan," Journal of Applied Sciences, vol. 2, no. 11, pp. 1016-1017, 2002.

[14] P. E. Bland, Rings and Their Modules, Walter de Gruyter \& Co., Berlin, Germany, 2011.

[15] T. Y. Lam, Lectures on Modules and Rings, Springer-Verlag, New York, NY, USA, 1998. 